\title{
Co-deformation of crystalline-amorphous nanolaminates
}

Wei Guo ${ }^{1,2}$, Jiahao Yao ${ }^{3}$, Eric A. Jägle ${ }^{1}$, Pyuck-Pa Choi ${ }^{1}$, Dierk Raabe ${ }^{1}$

${ }^{1 .}$ Department of Microstructural Physics and Alloy Design, Max Planck Institut für Eisenforschung, Düsseldorf, Germany

2. Microscopy Center for Nanophase Materials Science, Oak Ridge National Laboratory, Oak Ridge, USA.

3. Shenyang National Laboratory for Materials Science, Institute of Metal Research, Chinese Academy of Sciences, Shenyang, China

Deformation of ductile crystalline-amorphous nanolaminates is not clearly understood due to the complex interplay of interface mechanics, shear banding and deformation-driven chemical mixing. In this work, we synthesized and indented model nanolaminates consisting of nanocrystalline $\mathrm{Cu}$ and amorphous CuZr (Fig1a-c). In order to study both, nanostructural and atomic-scale chemical deformation effects at the same specimen position we performed a joint analysis by transmission electron microscopy (TEM) and Atom Probe Tomography (APT).

For the correlative TEM-APT analysis, tip-shaped specimens containing deformation shear bands were prepared by site-specific preparation, mounted onto an electro-polished bisected TEM $\mathrm{Cu}$ grid, and thinned by annular FIB milling. APT was performed with a local electrode atom probe (LEAP 3000X HR). Samples were analyzed at a base temperature of $60 \mathrm{~K}$, applying $532 \mathrm{~nm}$ wavelength 10 ps laser pulses of $0.4 \mathrm{~nJ}$ with at a repetition rate of $250 \mathrm{kHz}$. The datasets were reconstructed and analyzed using the software IVAS 3.6.6 (CAMECA Instruments).

Fig. 1 reveal zones with a large and very sharp, abruptly sheared $\mathrm{Cu}$ layer offset $>30 \mathrm{~nm}$. The layer is not completely disconnected from the sheared region though. Instead, it is heavily deformed at a $70.5^{\circ}$ tilt angle to the interface plane, which corresponds to the angle of the $\{111\}$ planes in fcc $\mathrm{Cu}$. In the shear band region, the $\mathrm{Cu}$ layers are subjected to a huge true shear strain of $4.0 \pm 0.2$, as calculated from the $\mathrm{Cu}$ layer displacement. The observations indicate dislocation slip on $\{111\}$ planes in the $\mathrm{Cu}$ layer. The large displacement $(>30 \mathrm{~nm})$ of the sheared and hence fragmented Cu layer shown in Fig. 1e suggests that large numbers of $\mathrm{Cu}$ dislocations accommodate the shear inside the crystalline phase, extending it across the $\mathrm{Cu} / \mathrm{CuZr}$ interface into the initially amorphous layer.

We have probed several compositional profiles across the $\mathrm{Cu}$ enriched region inside the shear band using small sampling volumes (size: $5 \times 5 \times 15 \mathrm{~nm}^{3}$ ). This analysis reveals that deformation-induced mixing in the sheared regions of the $\mathrm{Cu}$ layer is caused by the shear band running through it: In this mixing zone the $\mathrm{Cu}$ concentration varies from 64.6 at.\% to 95.4 at.\% within a narrow shear layer thickness range of only $2 \sim 3 \mathrm{~nm}$. Regions outside of the shear band have the same interface width and chemical concentration profiles as the as-deposited layers, i.e. they do not undergo deformation-driven mixing.

Fig. 2 shows TEM obtained from the sheared offset region of shear bands in the $\mathrm{CuZr} / \mathrm{Cu}$ multilayer specimen. NBD patterns taken along the shear band penetrating the initially amorphous $\mathrm{CuZr}$ layer show both, an inner halo ring (amorphous material) as well as some weak discrete diffraction spots 
(crystalline material) outside of the inner halo ring (see small arrows, NBD spots 3,4,6). This indicated that in the shear banded $\mathrm{Cu}$-enriched zones the initially amorphous $\mathrm{CuZr}$ forms an amorphous plus crystalline nanocomposite.

The present observation can explain serveral co-deformation and shear-induced mixing phenomena: (i) Local thinning of the $\mathrm{Cu}$ phase can be attributed to the high density of gliding dislocations. (ii) $\mathrm{Cu}$ atoms are dragged across the hetero-interface when dislocations release their shear step into the amorphous $\mathrm{CuZr}$ phase. The associated $\mathrm{Cu}$ enrichment can lead to local crystallization. (iii) Besides such dislocation driven solute mixing, nm-sized portions of crystalline $\mathrm{Cu}$ are in compact form displaced into the amorphous CuZr phase.

\section{References:}

[1] W Guo et al, Phys. Rev. Lett. 113 (2014) 035501.

[2] W Guo et al, Mater. Sci. Eng A. 628 (2015) 269-280.

[3] The authors acknowledge fruitful discussions with Dr. Daniel Haley and Dr. Michael Herbig.

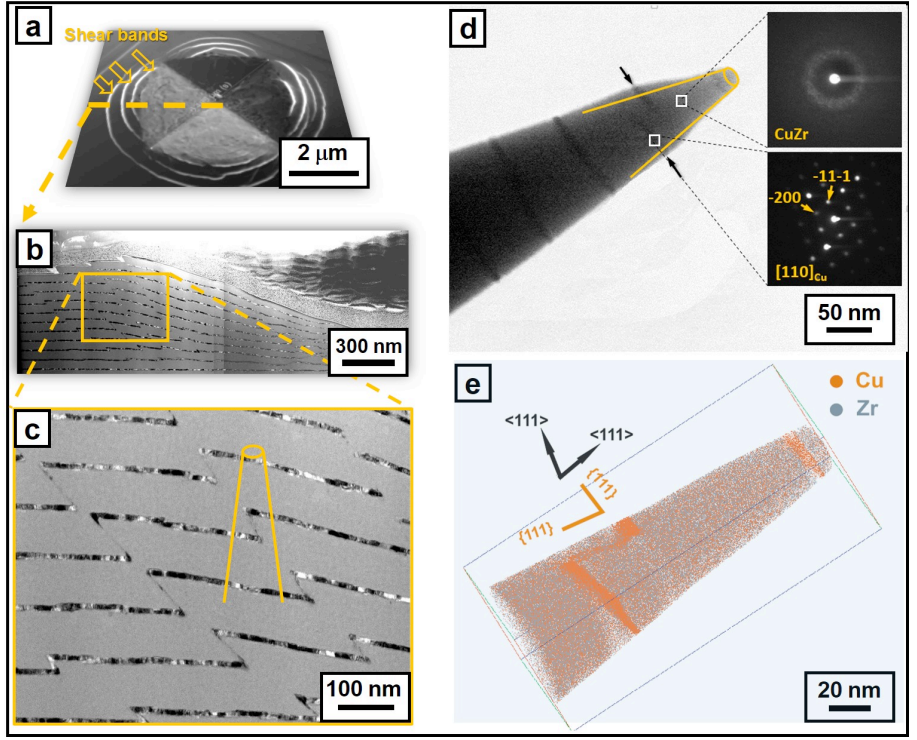

Figure 1. Correlative TEM/APT analysis of a shear band in amorphous $\mathrm{CuZr} /$ nanocrystalline $\mathrm{Cu}$ nanolaminates.

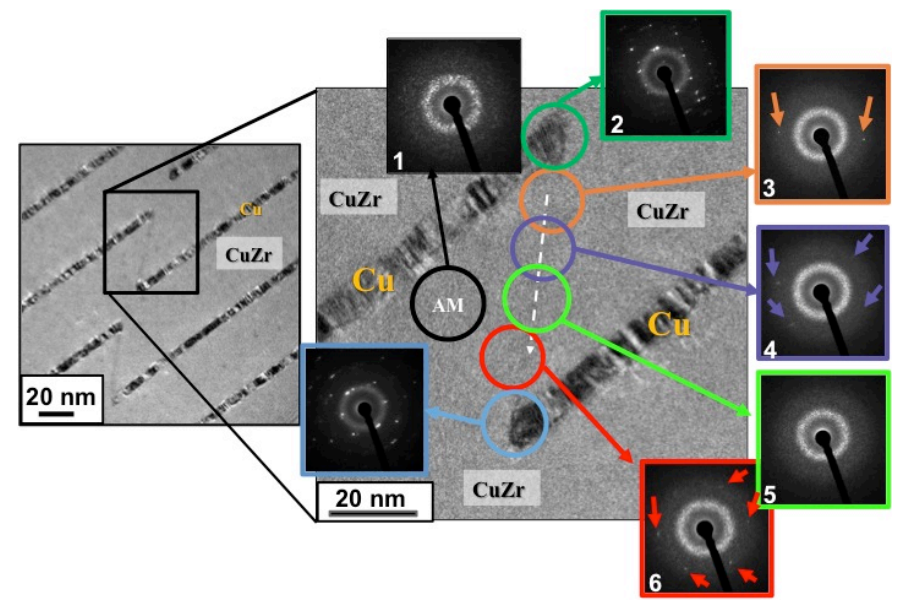

Figure 2. TEM observation of cross-phase shear bands in $100 \mathrm{~nm} \mathrm{CuZr} / 10 \mathrm{~nm} \mathrm{Cu}$ nanolaminates. 